\title{
Development of The American Association for Thoracic Surgery guidelines for low-dose computed tomography scans to screen for lung cancer in North America: Recommendations of The American Association for Thoracic Surgery Task Force for Lung Cancer Screening and Surveillance
}

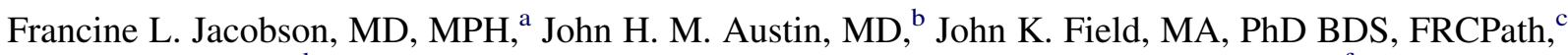 \\ James R. Jett, MD, ${ }^{\mathrm{d}}$ Shaf Keshavjee, MSc, FRCSC, MD, ${ }^{\mathrm{e}}$ Heber MacMahon, MB, BCh, ${ }^{\mathrm{f}}$ \\ James L. Mulshine, MD, ${ }^{\mathrm{g}}$ Reginald F. Munden, MD, DMD, MBA, ${ }^{\mathrm{h}}$ Ravi Salgia, MD, PhD, ${ }^{\mathrm{f}}$ \\ Gary M. Strauss, MD, ${ }^{i}$ David J. Sugarbaker, MD, ${ }^{\mathrm{a}}$ Scott J. Swanson, MD, ${ }^{\mathrm{j}}$ William D. Travis, MD, ${ }^{\mathrm{k}}$ and \\ Michael T. Jaklitsch, MD
}

Objective: The study objective was to establish The American Association for Thoracic Surgery (AATS) lung cancer screening guidelines for clinical practice.

\begin{abstract}
Methods: The AATS established the Lung Cancer Screening and Surveillance Task Force with multidisciplinary representation including 4 thoracic surgeons, 4 thoracic radiologists, 4 medical oncologists, 1 pulmonologist, 1 pathologist, and 1 epidemiologist. Members have engaged in interdisciplinary collaborations regarding lung cancer screening and clinical care of patients with, and at risk for, lung cancer. The task force reviewed the literature, including screening trials in the United States and Europe, and discussed local best clinical practices in the United States and Canada on 4 conference calls. A reference library supported the discussions and increased individual study across disciplines. The task force met to review the literature, state of clinical practice, and recommend consensus-based guidelines.
\end{abstract}

Results: Nine of 14 task force members were present at the meeting, and 3 participated by telephone. Two absent task force members were polled afterward. Six unanimous recommendations and supporting work-up algorithms were presented to the Council of the AATS at the 2012 annual meeting in San Francisco, California.

Conclusions: Annual lung cancer screening and surveillance with low-dose computed tomography is recommended for smokers and former smokers with a 30 pack-year history of smoking and long-term lung cancer survivors aged 55 to 79 years. Screening may begin at age 50 years with a 20 pack-year history of smoking and additional comorbidity that produces a cumulative risk of developing lung cancer of $5 \%$ or greater over the following 5 years. Screening should be undertaken with a subspecialty qualified interdisciplinary team. Patient risk calculator application and intersociety engagement will provide data needed to refine future lung cancer screening guidelines. (J Thorac Cardiovasc Surg 2012;144:25-32)

Lung cancer is the leading cause of cancer deaths in the United States and Canada. In 2012, the National Cancer Institute estimates that there will be 226,160 new cases diagnosed and 160,340 deaths in the United States. ${ }^{1}$ Ninety-four million current and former smokers in the United States remain at elevated risk for lung cancer, the leading cause of

\footnotetext{
From Brigham and Women's Hospital, ${ }^{\text {a }}$ Harvard Medical School, Boston, Mass; Columbia Presbyterian Hospital, ${ }^{\mathrm{b}}$ New York, NY; University of Liverpool, ${ }^{\mathrm{c}}$ Liverpool, England; National Jewish Hospital, ${ }^{\mathrm{d}}$ Denver, Colo; Toronto General Hospital, ${ }^{\mathrm{e}}$ Toronto, Ontario, Canada; University of Chicago, ${ }^{\mathrm{f}}$ Chicago, Ill; Rush Medical College, ${ }^{\mathrm{g}}$ Rush University, Chicago, Ill; The University of Texas MD Anderson Cancer Center, ${ }^{\mathrm{h}}$ Houston, Tex; Tufts Medical Center, ${ }^{\mathrm{i}}$ Boston, Mass; Dana-Farber Cancer Institute, ${ }^{\mathrm{j}}$ Boston, Mass; and Memorial Sloan-Kettering Cancer Center, ${ }^{\mathrm{k}}$ New York, NY.

Disclosures: John H. M. Austin, Reginald F. Munden, Gary M. Strauss, David J. Sugarbaker, William D. Travis, and Michael T. Jaklitsch have no commercial interests to disclose. Francine L. Jacobson reports grant funding from Toshiba. John K. Field reports advisory board member work for Epigenomics and Roche Diagnostics. James R. Jett reports grant research support from Oncimmune and Metabolomx. Shaf Keshavjee reports grant research support from Astellas Canada and
}

cancer death in the United States and Canada. In Canada, it is estimated that 25,600 new cases will be diagnosed and that 20,200 will die of the disease. ${ }^{2}$ The incidence of lung cancer increases with age, and consequently more than half of Americans and Canadians who are diagnosed with lung cancer are aged 70 years or more. Of note,

Axela/Exceed, clinical trial support from Vitrolife, and being awarded a Wyeth Pharmaceuticals/CIHR Rx\&D Clinical Research Chair in Transplantation. Heber MacMahon reports advisory board member and consultant work for Riverain Medical, consultant work for Biomet, and royalties from UC Tech (University of Chicago). James L. Mulshine reports a family member who is an employee of Accretive Health. Ravi Salgia reports advisory board member work for Cephalon and Methylgene, and research funding from Eli Lilly. Scott J. Swanson reports consulting fees for Ethicon and Covidien.

Address for reprints: Francine L. Jacobson, MD, MPH, Brigham and Women's Hospital, Department of Radiology, 75 Francis St, Boston, MA 02115 (E-mail: fjacobson@partners.org).

$0022-5223 / \$ 36.00$

Copyright (C) 2012 by The American Association for Thoracic Surgery doi:10.1016/j.jtcvs.2012.05.059 


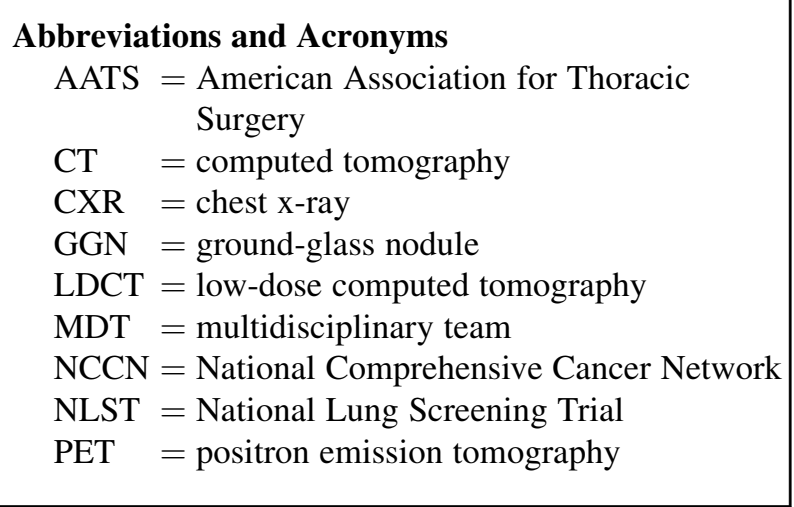

between 1996 and 2006, the incidence rates for those aged 75 years or more increased, whereas the overall incidence rates declined. ${ }^{3}$ Only $15.9 \%$ of those diagnosed with lung cancer survive 5 years, in part because of the association of advanced disease stage with initial symptoms of the disease. This is a lower survival than for cancers with current screening programs (breast, colon, and prostate).

An effective screening program that identifies early-stage disease before symptoms would have a powerful public health impact. Randomized trials for chest X-ray (CXR) and sputum cytology failed to establish a role for screening to detect early-stage lung cancer. The advent of single breath-hold volumetric computed tomography (CT) scanning of the chest at an acceptable level of radiation led to low-dose CT (LDCT) screening trials. The increased incidence of lung cancer coupled with increased therapeutic options and decreased rates of infectious diseases, such as tuberculosis, in the United States and Canada, also helped to increase the potential ability for screening to decrease lung cancer morbidity and mortality. The number of cases of lung cancer in the United States has nearly doubled since 1980 , when there were only 117,000 new cases. ${ }^{4}$ Tuberculosis peaked in 1993 with more than 26,000 reported cases in the United States, compared with 11,545 cases in $2009 .^{5}$ Minimally invasive thoracic surgery techniques pioneered in the 1990s now produce the remarkably low mortality rate found in prospective trials that involve lung cancer resection by lobectomy. ${ }^{6}$

Although demographics and technology have changed, popular opinions also have changed. The realization that tobacco companies deliberately added ingredients to cigarettes that may have increased their addictive potential, ${ }^{7}$ and the growing population of "never smokers" with lung cancer, especially among women, have changed the perception of lung cancer as a self-inflicted disease. ${ }^{8}$

\section{DEVELOPMENT AND SUCCESS OF LOW-DOSE COMPUTED TOMOGRAPHY}

These changes in our modern society led many researchers to believe that the time had come to reexamine methods to screen for early-stage lung cancer. CT scanning with the newer volumetric technology allowed radiologists to identify small nodules and parenchymal features and to study the outcome of patients with these findings in the course of nonrandomized studies in Japan and other countries. The Early Lung Cancer Action Project undertaken by Henschke and colleagues ${ }^{9}$ at the Weill-Cornell Medical Center studied this technology in 1000 patients who received both LDCT and CXR in a case-controlled study design. They consolidated the emerging knowledge about the imaging of early lung cancer findings that revolutionized the ability to detect disease before the onset of symptoms. The International Early Lung Cancer Action Program was started in 1993 and screened patients aged 60 years or more with a 10 pack-year smoking history. ${ }^{10}$ This single-arm study identified 484 lung cancers in 31,567 subjects. Eighty-five percent of the cancers were stage I, and the 10-year survival of these patients was $88 \%$. In contrast, 8 patients with stage I lung cancer who refused surgery had all died of disease within 5 years. Surgical mortality in this study was $0.05 \%$ when boardcertified thoracic surgeons in cancer centers provided the surgical care. The International Early Lung Cancer Action Program developed a robust care model; however, improved survival could not be used as a surrogate for decreased mortality.

The National Cancer Institute sponsored a randomized phase III National Lung Screening Trial (NLST) that, for the first time, provided level I evidence that LDCT screening can reduce lung cancer-specific mortality. Between August 2002 and April 2004, 53,454 individuals at high risk for lung cancer enrolled for 3 annual screens, with half receiving LDCT and half receiving CXR; all individuals then received follow-up through 2009 before early termination of the trial, when a $21 \%$ reduction in lung cancer-specific mortality was reached in the LDCT arm. ${ }^{11}$ This was achieved by a trial designed to have $90 \%$ power to detect this level of difference in the most cost-effective manner possible, using only 3 screens of a very high-risk population during the years leading up to the peak age range for lung cancer. The NLST enrolled smokers and former smokers, who quit smoking less than 15 years earlier, between 55 and 74 years of age with at least a 30 pack-year history of cigarette smoking. Subjects were excluded if they had a history of lung cancer, a CT scan of the chest within 18 months of enrollment, hemoptysis, or an unexplained weight loss more than $6.8 \mathrm{~kg}(15 \mathrm{lbs}){ }^{12}$

Three consecutive annual screens provided a prevalence screen and 2 annual screens. Longer duration of screening was not economically viable where each annual round of screen generated 26,723 LDCT scans and an equal number of CXR examinations. Positive screens were obtained in $24.2 \%$ of the LDCT arm and $6.9 \%$ of the CXR arm. Adverse events, including those from needle biopsy and 
surgical resection, occurred in $1.4 \%$ of those in the LDCT arm and $1.6 \%$ in the CXR arm.

The surgical mortality of less than $1 \%$ among those patients who underwent surgical resection of lung cancer is vital to the practical success of lung cancer screening in clinical practice. The NLST secondary analyses will continue to provide additional data and insight over the coming years, as will smaller prospective randomized trials accruing individuals to LDCT protocols in Europe. The NLST has the power to provide convincing evidence in favor of lung cancer screening at this time. Sufficient data are currently available to provide guidelines in favor of lung cancer screening in North America.

\section{MATERIALS AND METHODS}

With the announcement of mortality benefit for LDCT in July 2011, The American Association for Thoracic Surgery (AATS) councilors considered how best to translate the landmark work of the NLST into workable clinical guidelines for screening at-risk patients. In addition, thoracic surgeons sought to provide the dose-reduction benefits of LDCT to the more than 400,000 long-term lung cancer survivors in the United States and Canada. Co-chairs Francine Jacobson, MD, MPH, a thoracic radiologist, and Michael Jaklitsch, MD, a thoracic surgeon, were appointed and asked to create a committee with 12 to 20 members who would recommend lung cancer screening and surveillance guidelines to the Council by April 2012. The co-chairs created an interdisciplinary list of stakeholders whose academic careers have been dedicated to decreasing the morbidity and mortality of lung cancer through the early identification and successful treatment of the disease. The selection process was multifactorial and included individuals who have been involved in a wide variety of lung cancer and lung screening trials. Invitations were extended to more than 20 key stakeholders who were also invited to nominate others to serve on the task force, and 14 engaged with exceptional commitment. Task force members represent the specialties of thoracic surgery, pulmonary medicine, radiology, oncology, pathology, and epidemiology. Members also have related cross-disciplinary interests, and several have led key projects leading up to this milestone in the history of lung cancer, including various lung cancer screening projects over the past 20 years. Several members have equally strong credentials in at least one additional specialty, increasing perspective across disciplines with direct representation of surgery (4), pulmonary medicine (1), radiology (4), oncology (4), pathology (1), and epidemiology (1). The group provides care in a variety of settings in the United States and Canada. International participation was valued for perspective and coordination of intersociety dialog through the International Society for the Study of Lung Cancer.

\section{Charge to Committee}

In September 2011, the task force was charged to establish guidelines for lung cancer screening and surveillance of lung cancer survivors for development of second lung cancer in the North American population. By acknowledging that guidelines may differ for different populations, the group set out to develop detailed guidelines and algorithms for the identification, workup, and treatment of screen-identified lung cancer. The interdisciplinary organizational structure chosen for this task force was designed to support the unique central voice of the AATS and to serve other professional societies and the public. The task force was asked to structure their recommendations to be relevant for up to 7 to 10 years from now, when molecular epidemiology is likely to be established and biomarkers may play a more important role than LDCT. The dialog is thus about early identification of lung cancer through a multidisciplinary process that can dynamically adapt to rapidly changing and increasingly personalized delivery of health care within a structured framework.

\section{Committee Process}

Four conference calls were used to explore differences in local clinical practice patterns and to both collect and discuss the extensive and dynamically changing literature bearing on decisions regarding clinical implementation of LDCT in the United States and Canada. A 1-day meeting was then held in Boston to review the literature, discuss the implications, and formally vote on recommendations to present to the Councilors of the AATS. A system was prepared in advance to allow voice to significant dissenting opinions and rank strength of evidence separately from strength of recommendation. All meetings had agendas circulated beforehand and minutes circulated afterward. Initial conference calls were recorded to improve transcription of notes. The AATS provided access to selected literature for online review. Because of the dynamic nature of the literature in this field, the task force was able to include the National Comprehensive Cancer Network (NCCN) 2012 guidelines ${ }^{13}$ and an actuarial analysis of cost-benefit in preparing the task force recommendations. ${ }^{14}$ The task force used the process begun with the NCCN guidelines to broaden the recommendation for screening in the United States to extend the findings of the NLST as a research study to long-term clinical health care.

\section{RESULTS}

Six unanimous recommendations accompanied by the algorithms published as part of the AATS guidelines were presented to the Councilors of the AATS at the 2012 annual meeting. These 6 recommendations were accepted by the AATS (Table 1).

(1) Annual lung cancer screening with LDCT is recommended for smokers and former smokers with a 30 packyear history of smoking from age 55 to 79 years. This recommendation is based on NLST, age as an independent risk factor, and life expectancy. Furthermore, there is no scientific evidence to stop screening after 3 annual screens. The divergence of survival graphs in the NLST during the screening period followed by more parallel course of survival curves during the observation period suggests that the mortality reduction would have exceeded $20 \%$ if annual screening

TABLE 1. Recommendations of The American Association for Thoracic Surgery Task Force for Lung Cancer Screening and Surveillance in the United States and Canada

1. Annual lung cancer screening with LDCT for smokers and former smokers with 30 pack-year history of smoking from ages 55 to $79 \mathrm{y}$.

2. Long-term lung cancer survivors should have annual LDCT to detect second primary lung cancer until the age of $79 \mathrm{y}$.

3. Annual lung cancer screening with LDCT for smokers and former smokers aged 50 to $79 \mathrm{y}$ with a 20 pack-year history of smoking and additional comorbidity that produces a cumulative risk of developing lung cancer of $\geq 5 \%$ over the following $5 \mathrm{y}$.

4. Lung cancer screening and successful treatment of early-stage lung cancer by a subspecialty qualified team, including thoracic surgeons, thoracic radiologists, pulmonologists, oncologists, and pathologists.

5. Develop a web-based application for patient self-risk assessment.

6. Continue AATS engagement with other specialty societies to develop and refine future screening guidelines.

LDCT, Low-dose computed tomography; AATS, American Association for Thoracic Surgery. 
examinations had been continued. The task force therefore recommended increasing the upper age limit from 74 years, the median age for lung cancer incidence, to 79 years based on the life expectancy at age 74 years, on average in the United States, of 78.6 years. Thus, screening should add 7 years to life expectancy. It is possible that the extremely elderly represent a different population who may warrant individual case consideration for screening based on health and functional status. Screening should not be offered to individuals for whom adequate treatment cannot be offered because of comorbidity or functional status, regardless of age.

(2) Individuals treated for lung cancer should receive surveillance for recurrent disease and for new lung cancer. The initial surveillance beyond the perioperative period is designed primarily to identify recurrent disease. By 4 to 5 years after therapy, the purpose of CT follow-up is to provide surveillance to detect new primary lung cancer. Therefore, these patients should have annual LDCT to detect new primary lung cancer until the age of 79 years.

(3) The incorporation of smaller risk factors will become more objective over the next several years. The NCCN guidelines provide a second-tier recommendation for the addition of a risk factor such as chronic obstructive pulmonary disorder combined with younger age and less smoking history. The task force strongly encourages the use of risk calculators to assist in creating a more quantified approach to this extension. Beginning annual lung cancer screening with LDCT for smokers and former smokers at age 50 years with a 20 pack-year history of smoking should be considered when the additional comorbidity produces a cumulative risk of developing lung cancer of $5 \%$ or more over the following 5 years. Screening of a woman may be deferred before menopause.

(4) Realizing the potential for lung cancer screening and successful treatment of early-stage lung cancer by a multidisciplinary team (MDT) requires active participation by subspecialty-qualified thoracic surgeons, thoracic radiologists, pulmonologists, oncologists, and pathologists. The perspective of each specialty is required to establish and maintain a local implementation of lung cancer screening guidelines. The sharing of vocabulary, emerging literature, and patient outcomes across specialties is critical for smooth functioning and further development of a screening program. Thoracic surgeons trained in minimally invasive capabilities, such as video-assisted thoracoscopic surgery, will maintain the low operative mortality for invasive procedures. We further recommend that volumetric analysis be used, when possible, to guide decisions.

(5) The task force recommends the development of a web-based application for handheld devices to allow patients to access educational information, assess personal risk of lung cancer, and potentially allow voluntary anonymous data collection about the population risk of lung cancer in the United States and Canada. This approach would support the quantitative approach recommended for combining risks in a manner that can be updated easily as more data bearing on such decisions become available.

(6) The AATS has established engagement across specialties that needs to be maintained to pursue the research that is still needed to answer the myriad of questions that have not been answered and those that have not been asked regarding lung cancer screening. Through ongoing engagement with other specialty societies and International Society for the Study of Lung Cancer, the AATS will maintain the central role for thoracic surgeons in the diagnosis and treatment of lung cancer.

\section{Clinical Lung Cancer Screening}

The task force identified more similarities than differences between different trials and different communities in the United States and Canada. The following material provides practical strategies intended to serve as starting points for local implementation guided by the local MDT. The MDT may mirror the task force membership with participation by the specialists regularly involved in the diagnosis and treatment of early lung cancer in the community. Representation should include subspecialty-trained radiologists, surgeons, internists, and pathologists who provide patient care and follow the scholarly progress regarding lung cancer screening within their specific disciplines. Regular conferences allow for input from additional perspectives and encourage the maintenance and dissemination of outcomes within local communities. The MDT is most able to maximize smoking cessation within the context of lung cancer screening and interface with grass-roots advocacy groups and professional societies. This structure will minimize the time required to implement changes that will evolve through emerging data analysis and development of alternative screening methods based on biomarkers.

\section{Type of Scan}

LDCT should be performed using multidetector CT scan technology with the lowest dose that will provide adequate visualization and measurement of small lung nodules (average effective dose in the NLST was $1.5 \mathrm{mSv}$ ). The NLST scanning protocol is technologically feasible in general community settings. All machines should meet the technical standards of the American College of Radiology. Dose modulation may be used. Subcentimeter slices are desirable for measurement by volumetric software when possible; however, 1- to 2-mm contiguous images are acceptable.

The value to patient health from LDCT is broader than just the identification of lung cancer. Emphysema and other lung diseases should be assessed. Coronary calcifications are clearly seen on most scans and should be reported. Incidental findings that have contributed to the all-cause mortality benefit found by NLST include renal cell carcinomas and aortic aneurysms. 


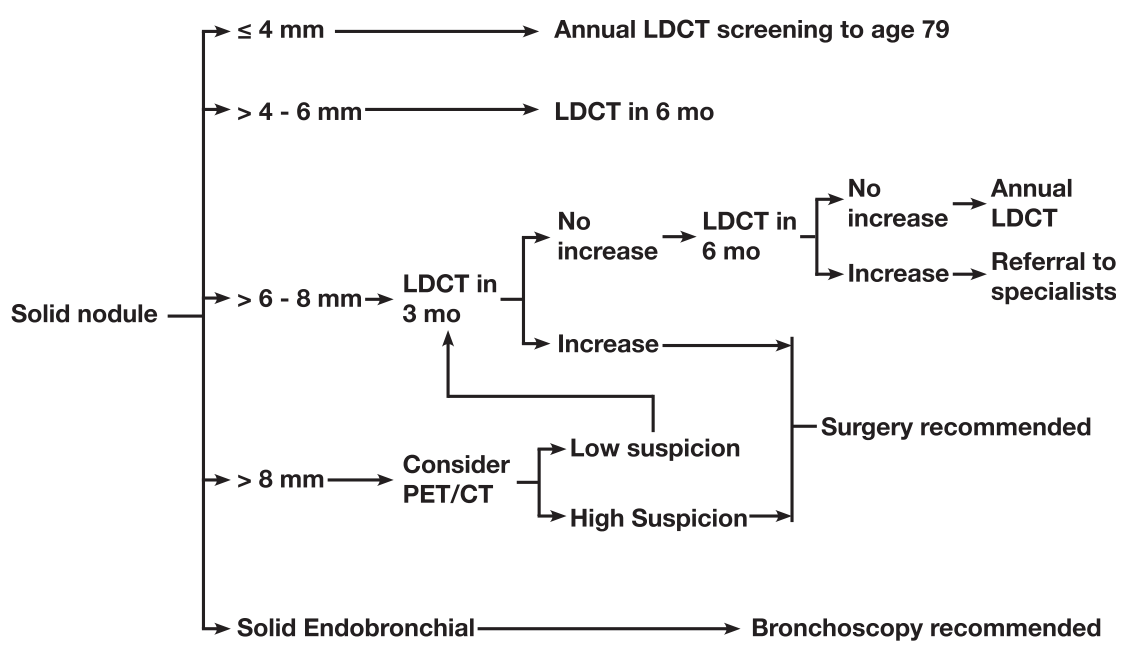

FIGURE 1. AATS lung cancer screening guidelines for solid nodules on low-dose computed tomography (LDCT). PET/CT, Positron emission tomography/ computed tomography.

\section{Management of Indeterminate Solid Nodules}

Indeterminate solid nodules are managed by size (Figure 1). Baseline LDCT is negative if no nodules are present. It is also negative when only solid nodules $4 \mathrm{~mm}$ or less in diameter are present. Tiny solid nodules are frequent findings and unlikely to represent early lung cancer, particularly if they do not increase in size between the prevalence and first incidence screens. These individuals should continue with routine annual LDCT screening until age 79 years.

Solid nodules with a mean diameter between 4 and $6 \mathrm{~mm}$ are considered indeterminate and should have a repeat LDCT in 6 months. In the case of stable or smaller nodule(s), a screen is then considered negative, and these individuals should then continue with annual LDCT screening up to age 79 years.

Solid nodules with a mean diameter of 6 to $8 \mathrm{~mm}$ also should be considered indeterminate with a repeat LDCT in 3 months. If the nodule remains solid and decreases in size, the screen is reclassified as negative and the individual should return to annual LDCT screening up to age 79 years. A stable nodule in this size range (mean diameter $>6-8$ $\mathrm{mm}$ ) is still considered an indeterminate nodule and should have a repeat LDCT in 6 to 9 months ( 9 months coincides with annual screen). If the subsequent LDCT scan reveals a decrease in diameter or stable mean diameter on the second scan, the screen is classified as a negative result and the patient should return to annual LDCT up to age 79 years. If there is a further increase in the nodule on the second LDCT, this is considered a positive screen with consideration of biopsy and treatment (preferably surgical removal).

Solid nodules with a mean diameter greater than $8 \mathrm{~mm}$ are classified as screen positive and should lead to referral to the specialists who oversee the workup and management of lung cancer in the community. A positron emission tomography (PET)/CT scan may obviate the need for surgery if the scan suggests a low suspicion of malignancy. In the face of such a low suspicion due to a PET/CT scan, however, a repeat LDCT scan should be obtained in 3 months, and increase in size or geometry should then be interpreted as a positive screen with surgical intervention. If no PET/ CT is obtained, or a PET/CT is suspicious for malignancy, the patient should be referred for surgical staging and resection. The finding of a solid endobronchial lesion on an LDCT scan should lead to referral to a specialist for bronchoscopy.

\section{Ground-Glass Nodules}

Ground-glass nodules (GGNs) are well-demarcated nodules of subtly increased attenuation through which vessels can still be separately visualized that require a separate algorithm for workup (Figure 2). GGNs are also sometimes described as nonsolid nodules. Homogeneous GGN may represent atypical adenomatous hyperplasia when $5 \mathrm{~mm}$ or less and adenocarcinoma in situ when larger. In the adenocarcinoma disease spectrum, the development of solid components increases concern for invasive malignancy. These most worrisome nodules may be referred to as part-solid. The determination of worrisome changes in such nodules is complex because of potential changes that may increase or decrease overall size of a GGN and size of solid component. Increasing density alone may indicate progressive disease.

Inflammatory processes can also cause ground-glass opacities. Early pneumonia and pneumonia in immunecompromised hosts may have ill-defined ground-glass opacities with or without areas of more solid-appearing opacity and consolidation.

The AATS recommends consistent application of size guidelines, such as those developed by the Fleischner Society, for management of ground-glass opacities discovered on LDCT at baseline scan or during an annual LDCT screening examination within the previously described screening populations. 


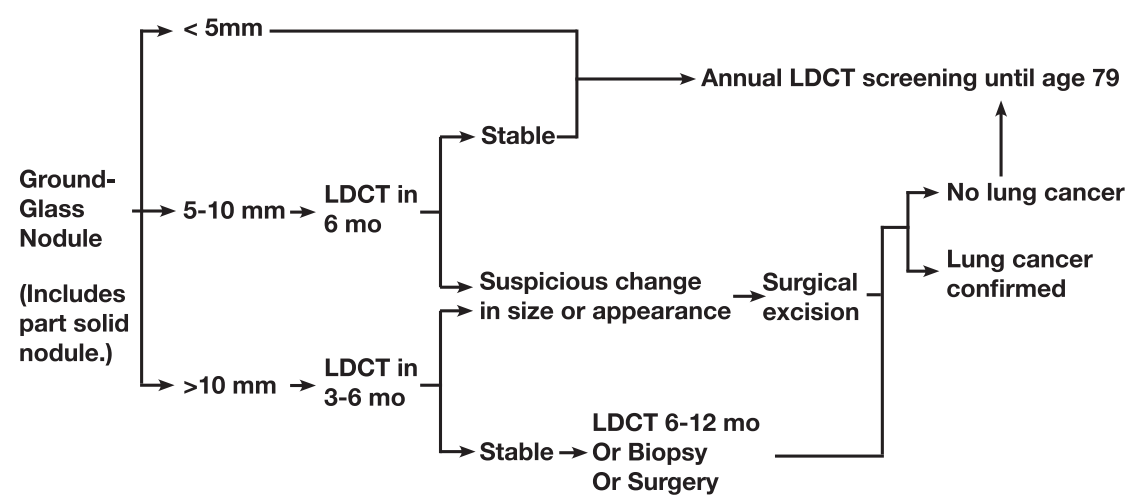

FIGURE 2. AATS lung cancer screening guidelines for ground-glass nodule. $L D C T$, Low-dose computed tomography.

An increase in the size of a GGN that was originally less than $5 \mathrm{~mm}$ or a change in a GGN such as the development of a solid component should increase consideration of surgical resection. If surgical resection is not practical for a particular small changing GGN, a repeat LDCT should be obtained in 3 to 6 months. If the lesion is stable, continued screening with annual LDCT should be resumed up to age 79 years for high-risk populations. If the repeat LDCT scan shows continued growth, biopsy or surgical excision may become feasible.

For GGNs between 5 and $10 \mathrm{~mm}$ on baseline LDCT, a repeat LDCT should be performed in 6 months. Stable abnormalities would then revert to annual LDCT scans, whereas an increase in size or development of a solid component should lead to referral to a lung cancer specialist and consideration of surgical excision.

GGNs greater than $10 \mathrm{~mm}$ in mean diameter should have a repeat LDCT scan in 3 to 6 months, if not resected. If the lesion is considered stable, and not regressing in size, considerations include biopsy, surgical excision, and repeat LDCT in 6 to 12 months. GGNs greater than $10 \mathrm{~mm}$ in mean diameter that increase in size or develop a partsolid component on repeat scan should undergo surgical excision.

\section{Workup of Positive Annual Low-Dose Computed Tomography Scans}

If a new nodule is detected on an annual LDCT screen, further workup depends on the character and size of the lesion, and suspicion of infection (Figure 3).

If a new solid nodule does not show benign characteristics, then size mandates further workup and the algorithm presented in Figure 1 should be followed. The probability of malignancy in a new GGN without suspicion for infection also depends on size and should be worked up following the algorithm presented in Figure 2.

New nodules detected by LDCT scan that may be due to infection or inflammation should be treated with antimicrobials and then have a repeat LDCT scan in 1 to 2 months. If the inflammatory process is resolving, but a component remains, then repeat radiographic imaging should continue until complete resolution of this inflammatory process.

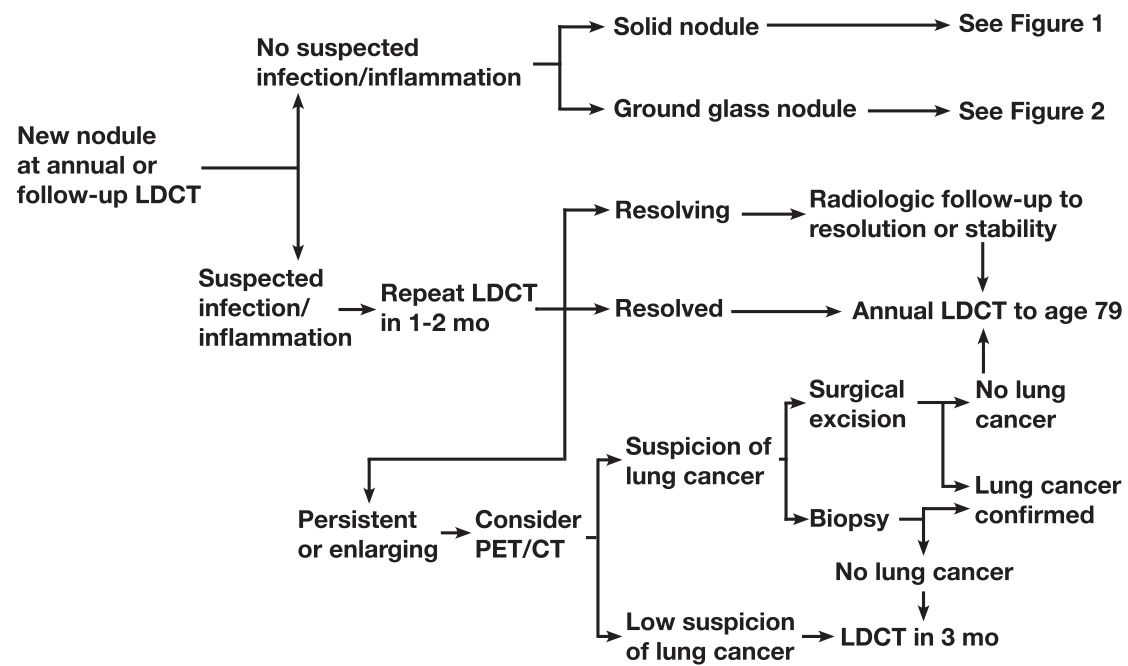

FIGURE 3. AATS lung cancer screening guidelines for new nodule on low-dose computed tomography (LDCT). PET/CT, Positron emission tomography/ computed tomography. 
High-risk populations with resolved lung inflammation should return to annual LDCT screening up to age 79 years.

GGN or other potentially inflammatory-appearing opacity on LDCT scan requires follow-up LDCT within 3 months; if the nodule increases in size, the screen is considered positive. A PET/CT scan should be considered, and surgery may be obviated by low suspicion of cancer on that scan, although adenocarcinoma can have little or no fluorodeoxyglucose avidity. An LDCT scan should be obtained in 3 months after such PET/CT scan. Further enlargement of the nodule on LDCT is considered a positive screen and warrants surgical excision. A CT scan obtained for attenuation correction of PET scan provides a significantly lowerquality scan than LDCT. Low-level fluorodeoxyglucose avidity is characteristic of both inflammation and relatively indolent adenocarcinoma, limiting its usefulness for differentiation of benign and malignant diseases.

\section{DISCUSSION}

Reducing the radiation dose for $\mathrm{CT}$ scanning has become a central focus for scanner manufacturers and radiologists. It is likely that in the near future, new methods of reconstructing the images will provide significantly lower doses than even the 1.5 total body equivalent dose estimates for NLST. Over the decade since the NLST opened, slice thicknesses and scan times have decreased further. Population screening using thin-section LDCT requires a laborintensive review process by the radiologist. Tiny nodules, below the threshold of a positive screen by size $(<4 \mathrm{~mm}$ in diameter), may be inconsistently imaged and inconsistently identified, particularly when multiple tiny nodules are present. The subjective and semiquantitative measurement of tiny nodules by radiologists using eye and electronic calipers varies by more than one doubling time between 4 and $5 \mathrm{~mm}$. Part-solid nodules and GGNs also pose challenges in the assessment of growth with critical implications for clinical decision-making. Volumetric nodule measurements are to be preferred and should be provided when possible. As this and computer-aided diagnosis become more widely available, they should be incorporated into the work-flow for routine interpretation of LDCT. Standardization of reporting, as done with Breast Imaging-Reporting and Data System (BIRADS) for mammography, also deserves encouragement.

\section{Radiation Risk}

The radiation exposure from LDCT is an acceptably small risk for high-risk populations. The average effective dose per scan in the NLST trial was $1.5 \mathrm{mSv} .{ }^{11}$ The American Association of Physicists in Medicine December 13, 2011, position statement on radiation risks from medical imaging procedures states, "Risks of medical imaging at effective doses below $50 \mathrm{mSv}$ for single procedures or 100 $\mathrm{mSv}$ for multiple procedures over short time periods are too low to be detectable, and may be nonexistent." 15

\section{Patient Requirements for Screening}

Approximately 7 million of 94 million current and former smokers in the United States meet the NLST criteria for highest risk screening. ${ }^{13}$ More than $75 \%$ of the positive screening tests resulted in additional testing, most frequently by repeat imaging. Less than $10 \%$ of the tests required an invasive procedure for further workup.

Screening should not be performed for individuals at elevated risk who do not have the physical reserve required for appropriate treatment. Intervention risks are related to performance status, and long-term survival is negatively affected by poor constitution. Performance status and lung function will be used to determine on an individual basis when to stop lung cancer screening. In the future, the use of ablation for early lung cancer may increase treatment potential for elderly patients. Extremely elderly patients who are well may also benefit from continued screening beyond age 79 years on an individual basis.

\section{Cost-Effectiveness of Screening}

In the NLST trial, 320 screens were necessary to detect 1 cancer. In other words, $\$ 35,000$ can detect and treat the single cancer, and reassure 319 others that they do not have lung cancer despite their pretest risk.

The most recent estimate of the cost and benefit of annual lung cancer screening offered as a commercial insurance benefit for high-risk Americans aged 50 to 64 years found a screening cost of $\$ 1$ per insured member per month in 2012 dollars. ${ }^{14}$ Furthermore, the cost per life-year saved would be less than $\$ 19,000$. This compares favorably to cost per life-year saved in breast $(\$ 31,000-\$ 52,000)$, colon $(\$ 19,000-\$ 29,000)$, and cervical cancers $(\$ 50,000$ $\$ 75,000$ ). The authors concluded that lung cancer screening for high-risk patients aged 50 years and more with 30 packyears smoking was high-value coverage.

\section{Future Directions}

In addition to the $20 \%$ decrease in lung cancer-specific mortality, the NLST found a 7\% decrease in all-cause mortality. From the perspective of the individual patient being cared for by his or her physician, LDCT provides additional valuable information regarding health and disease processes, including diffuse lung diseases and cardiovascular disease. Emphysema, identified by screening, is an independent risk factor for the development of lung cancer. As phenotypes of chronic obstructive pulmonary disorder are identified by the COPDGene study, it is reasonable to expect greater understanding of why particular patients develop lung cancer while others with the same smoking history do not. In the future, this may lead to differences in recommendations for screening based on individualized risk. The clinical LDCT goal is the early identification of lung cancer that enables successful treatment with the least morbidity and risk of mortality for the individual patient. In the future, we anticipate the 
use of a biomarker in urine or blood as the primary population screen. It may remain valuable to obtain a single baseline reference LDCT scan to validate the initial negative biomarker and serve as a comparison CT in the case of a subsequent positive screening test result.

Volumetric analysis may be a more accurate way to radiographically sort suspicious from nonsuspicious nodules. This technology is currently being used in the DutchBelgium Lung Cancer Screening Trial (NELSON). Measurements are performed using a volumetric analysis with a lower limit of $50 \mathrm{~mm}^{3}$ (4.6 mm diameter) identifying "negative" nodules. A "positive" nodule is any finding greater than $500 \mathrm{~mm}^{3}$ (>9.8 mm diameter). Between these 2 parameters, nodules are considered indeterminate, and repeat volumetric studies are particularly useful at identifying rapidly growing nodules that are then considered "positive." This may prove useful in the near future, although the penetrance of this technology into the community is not yet sufficient to be strongly advocated by the AATS at this time.

Prediction models of risk of developing lung cancer over the next 5 years are currently available: the Bach model based on data from Beta-Carotene and Retinol Efficacy Trial, the Spitz model based on data from an ongoing case-control study, and the Liverpool Lung Project based on a case-control study in Liverpool, England. At this time, these do not provide complete data for important subpopulations in North America. It is probable that a clinical prediction rule could be converted to a smart phone "App" that would allow patients to calculate their risk of developing lung cancer. This would be a way to enable individual patients to assess their own risk and to identify populations who would most benefit from screening. For now, these models lack sufficient accuracy for routine use but are expected to be refined with additional data collection.

It is highly likely that LDCT will be augmented or replaced as the primary lung cancer screening modality by a biomarker test, such as sputum, urine or blood, or even breath condensate as a potential primary population screen. It may remain valuable to obtain a single baseline reference CT scan to validate the initial negative biomarker and serve as a comparison $\mathrm{CT}$ in the case of a subsequent positive screening test result.

\section{Biomarkers}

There have been a number of serum biomarkers (from traditional carcinoembryonic antigen/cancer antigen 125 to microRNA) that have been used for potential screening without a large population validation. Methylation of certain genes, microRNA, and glycosylation of proteins have been detected in sputum and urine. A number of samples have been collected within the NLST, and several biomarkers are being analyzed within this study with future publications expected that may refine the screening method. To positively affect screening strategies, we need to be able to determine not one particular sample or pathway, but a global and systematic approach.

\section{CONCLUSIONS}

New data analyses will continue to update the view of professionals actively engaged in this dialog over the coming months and years. The issues that direct public health and societal allocation of resources will be important but we have entered a new era for the early detection of lung cancer. The recommendations of the AATS task force are offered with the intent to support individualized decision making based on the literature and best practices for the early diagnosis and treatment of lung cancer in the United States and Canada.

We would like to acknowledge Matt Eaton, Director of Administration, The American Association for Thoracic Surgery, for his invaluable assistance and Mark Jacobson for graphics.

\section{References}

1. American Cancer Society. Cancer Facts \& Figures 2012. Atlanta, GA: American Cancer Society; 2012.

2. Canadian Cancer Society's Steering Committee. Canadian Cancer Statistics 2009. Toronto: Canadian Cancer Society; 2009. Available at: http://www. cancer.ca/Canada-wide/About $\% 20$ cancer/Cancer $\% 20$ statistics/ $/$ media/CCS/ Canada \%20wide/Files\%20List/English\%20files\%20heading/pdf \%20not \%20 in $\% 20$ publications $\% 20$ section/Stats $\% 202009 \mathrm{E} \% 20 \mathrm{Cdn} \% 20 \mathrm{Cancer}$ ashx. Accessed May 28, 2012.

3. Canadian Partnership Against Cancer. System Performance Report 2010. Toronto: Canadian Partnership Against Cancer; 2010.

4. Strauss GM, Gleason RE, Sugarbaker DJ. Chest x-ray screening improves outcome in lung cancer: a reappraisal of randomized trials on lung cancer screening. Chest. 1995;107:270S-9S.

5. Pratt R, Robison V, Navin T. Trends in tuberculosis. MMWR. 2011;60;333-7. Centers for Disease Control and Prevention, March 25, 2011. Available at: http://www.cdc.gov/mmwr/pdf/wk/mm6011.pdf. Accessed May 28, 2012.

6. Allen MS, Darling GE, Pechet TT, Mitchell JD, Herndon JE II, Landreneau RJ, et al. Morbidity and mortality of major pulmonary resections in patients with early-stage lung cancer: initial results of the randomized, prospective ACOSOG Z0030 trial. Ann Thorac Surg. 2006;81:1013-20.

7. Wilkenfeld J, Henningfield J, Slade J, Burns D, Pinney J. It's time for a change: cigarette smokers deserve meaningful information about their cigarettes. $\mathrm{J}$ Natl Cancer Inst. 2000;92:90-2.

8. Jemal A, Chu KC, Tarone RE. Recent trends in lung cancer mortality in the United States. J Natl Cancer Inst. 2001;93:277-83.

9. Henschke CI, McCauley DI, Yankelevitz DF, Naidich DP, McGuinness G, Miettinen OS, et al. Early Lung Cancer Action Project: overall design and findings from baseline screening. Lancet. 1999;354:99-105.

10. Henschke CI, Yankelevitz DF, Libby DM, Pasmantier MW, Smith JP, Miettinen OS. Survival of patients with stage I lung cancer detected on CT screening. N Engl J Med. 2006;355:1763-71.

11. Aberle DR, Adams AM, Berg CD, Black WC, Clapp JD, Fagerstrom RM, et al. Reduced lung-cancer mortality with low-dose computed tomographic screening. N Engl J Med. 2011;365:395-409.

12. National Lung Screening Trial Research Team, Aberle DR, Berg CD, Black WC, Church TR, Fagerstrom RM, Galen B, et al. The National Lung Screening Trial: overview and study design. Radiology. 2011;258:243-53.

13. National Comprehensive Cancer Network Clinical Practice Guidelines in Oncology. Lung Cancer Screening Version 1.2012, October 26, 2011. Available at: NCCN.org. Accessed March 26, 2012.

14. Pyenson BS, Sander MS, Jiang Y, Kahn H, Mulshine JL. An actuarial analysis shows that offering lung cancer screening as an insurance benefit would save lives at relatively low cost. Health Aff. 2012;31:770-9.

15. American Association of Physicists in Medicine. PP 25-A: AAPM Position Statement on Radiation Risks from Medical Imaging Procedures. Available at: http://www.aapm.org/org/policies/details.asp?id=318\&type=PP\&lcurrent=true. Accessed May 28, 2012. 